\title{
16 New Therapeutics Targets in Chronic Viral Cardiomyopathy
}

W. Poller, H. Fechner, U. Kühl, M. Pauschinger, H.-P. Schultheiss

16.1 Introduction . . . . . . . . . . . . . . . . . 288

16.2 Virus-Host Interactions and Antiviral Therapy . . . . . . . . . . . 289

16.2.1 Therapeutic Targets (I): Virus Uptake Mechanisms . . . . . . . . . 295

16.2.2 Therapeutic Targets (II): Signaling Pathways of Innate Immunity . 297

16.2.3 Therapeutic Targets (III): Cellular Damage

by Virus-Encoded Proteins ． . . . . . . . . . . . . . . . . . . . . 298

16.3 Summary and Perspective . . . . . . . . . . . . . . . . . . . . . 299

References . . . . . . . . . . . . . . . . . . . . 300

Abstract. Dilated cardiomyopathy (DCM) is a prevalent heart muscle disease characterized by impaired contractility and dilation of the ventricles. Recent clinical research suggests that cardiotropic viruses are important environmental pathogenic factors in human DCM, which may therefore be considered as a chronic viral cardiomyopathy. All virus-positive DCM patients thus come into the focus of virological research and should be considered for antiviral strategies. Interferon- $\beta$ therapy has been shown to mediate virus elimination in patients with adenovirus or coxsackievirus persistence. We discuss here several possible new molecular targets for patients infected with cardiotropic viruses in (1) the cellular virus uptake system, (2) virus-induced cellular signaling pathways, and (3) interactions between virus-encoded proteins with important cellular target proteins. The potential of these approaches in the setting of a chronic viral infection is significantly different from that in an acute viral infection. Specific problems encountered in a chronic situation and possible solutions are discussed. 


\subsection{Introduction}

Dilated cardiomyopathy (DCM) is a prevalent heart muscle disease characterized by impaired contractility and dilation of the ventricles (Poller et al. 2005; Chien 2003; Franz et al. 2001). Recent clinical research suggests that cardiotropic viruses are important environmental pathogenic factors in human DCM, which may thus be considered as a chronic viral cardiomyopathy (Bowles et al. 1986; Kühl et al. 2003a, 2005; Pauschinger et al. 1999a,b; Why et al. 1994; Frustaci et al. 2003; Matsumori et al. 1995, 2000). Since DCM is a far more frequent disorder than acute viral cardiomyopathy (i.e., classical myocarditis), the new data on viruses in DCM hearts greatly expand the number of patients classified as having a virus-associated cardiac disease. All these patients therefore come into the focus of virological research and may be considered for antiviral strategies. In the following review, we discuss several novel therapeutic approaches derived from this broadened perspective on virus-associated cardiac disorders. Clinical endpoints for any new therapy of this type are improvements of symptoms or survival (Kühl et al. 2003a). Cardiac functional improvement may be expected:

1. If the virus-infected cardiac cells were eliminated by an antiviral immune response without over-shooting inflammation (Kühl et al. 2003a).

2. If intracardiac viruses were functionally "silenced" in the sense that no new virus particles or viral genomes are synthesized, and furthermore, that any transcriptional activity of the viral genomes is suppressed. If a major fraction of the cardiac cells were infected, such "silencing" may be preferable over immune elimination, i.e., destruction of infected cells.

3. If de novo infection of cardiac cells by virus spreading within the heart or from extracardiac virus pools would occur, blockade of this process at the receptor level may be useful. Possible new molecular targets to achieve the goals are located (1) in the cellular virus uptake machinery, in particular in the heart (Bergelson et al. 1997; Gaggar et al. 2003; Noutsias et al. 2001; Fechner et al. 2003; Dörner et al. 2004), (2) in virus-induced cellular signal pathways of the innate immune system (Ulevitch 2004; Hertzog et al. 2003), or (3) in interac- 
tions between virus-encoded proteins with structurally or functionally important target cell proteins.

The relative therapeutic potential of these targets is discussed while considering differences between acute vs chronic disease phases of a viral disorder, and between a human disease (Why et al. 1994; Baboonian et al. 1997) vs more or less analogous animal models of that disease (Yanagawa et al. 2004; Yanagawa et al. 2003). In contrast to some highly specialized animal models, very little is known so far in humans on the kinetics of the cardiac virus load, on possible migration within the heart or between cardiac and other, e.g., lymphatic cells, or on cellular signal pathway activation by the different cardiotropic viruses detected in human hearts. Certain therapeutic strategies suggested by cell biological (Dörner et al. 2004) or animal studies (Yanagawa et al. 2003, 2004) of coxsackievirus cardiomyopathy may possibly also work in humans, but for most cardiotropic viruses recently identified in human hearts (e.g., PVB19, HHV6, AdV) (Kühl et al. 2005), we do not have adequate animal models at the present time.

\subsection{Virus-Host Interactions and Antiviral Therapy}

1. Figure 1 provides a general overview on key interactions sites between viruses and their target cells. Most known cardiotropic viruses are taken up into the cell by receptor-mediated endocytosis (Bergelson et al. 1997; Gaggar et al. 2003; Noutsias et al. 2001; Fechner et al. 2003) and then further transported into the nucleus via the nuclear pore complex. The receptor level thus constitutes a first level of therapeutic targets (Fig. 2). If significant de novo infection of cardiac cells by virus spreading within the heart or from extracardiac virus pools occurs, therapeutic intervention at this level may be effective. Acute cardiac viral infections may not only be caused by CVB3 or AdV, but also by PVB19 (Kühl et al. 2003b; Rohayem et al. 2001; Nigro et al. 2000; Murry et al. 2001). In these often fulminant and ultimately fatal diseases, intervention at the receptor level may have particular therapeutic potential.

2. During the process of endocytosis, viral genomic DNA or RNA may activate signaling cascades of innate antiviral immunity via Toll-like 
receptors (TLR). On one hand this contributes to immunological elimination of virus-infected cells, on the other it may cause damage by overshooting immune reactions. Innate immune signaling thus constitutes a second level of therapeutic targets (Fig. 3) (Ulevitch 2004; Hertzog et al. 2003). An optimal antiviral immune response would pinpoint infected cells without collateral damage by inadequate inflammation. Virus elimination solicited by interferon (INF)$\beta$ treatment has already been shown to result in improved cardiac function and New York Heart Association (NYHA) classification in humans (Kühl et al. 2003a). Even if immune elimination of infected cells is not achieved, the suppression of inflammatory damage by

Fig. 1. Virus-host interactions and antiviral therapy. The frequencies at which cardiotropic viruses were detected in a series of $245 \mathrm{DCM}$ patients are parvovirus B19 (PVB19) (51.4\%), human herpes virus (HHV)6 (21.6\%), enteroviruses $(E V)$ (9.4\%), Epstein-Barr virus $(E B V)(2.0 \%)$, adenovirus (AdV) (1.6\%), and hepatitis $\mathrm{C}$ virus $(\mathrm{HCV})(0.8 \%)$. Also screened but not detected were HSV1/2, influenza (Inf)A/B, cytomegalovirus (CMV). Cellular entry of these viruses occurs via cell surface receptors and coreceptors, followed by receptor-mediated endocytosis of the virus particles, release of the viral RNA (CVB3) or DNA genomes (other viruses), and their nuclear important via the nuclear pore complex (NPC). Virus receptors form a first level for antiviral therapeutic interventions (see Fig. 2). Coxsackievirus-adenovirus receptor (CAR) shows high interindividual variability in human hearts (Noutsias et al. 2001; Fechner et al. 2003), but little is known on the cardiac expression of the other known receptors for cardiotropic viruses. Toll-like receptors (TLRs) 3,7,8,9 are located in the membrane of the endosomes and may trigger signaling cascades of innate antiviral immunity (INF- $\beta$ dependent and independent). TLRs and TLR-dependent signal pathways form a second level for therapeutic interventions (see Fig. 3). Knowledge on innate immune signal molecules in human hearts is sparse. When the viral genomes are transcribed in the nucleus and translated in the cytosol, their products may act upon and damage structural and functional proteins of the cardiomyocyte. Whereas for most human cardiotropic virus-encoded proteins no data on interactions with structural and functional proteins are available, dystrophin has been shown to be markedly vulnerable to cleavage by enteroviral protease $2 \mathrm{~A}$ (Badorff et al. 1999). Suppression of potentially damaging virus-encoded proteins may have therapeutic potential (Fig. 4) 


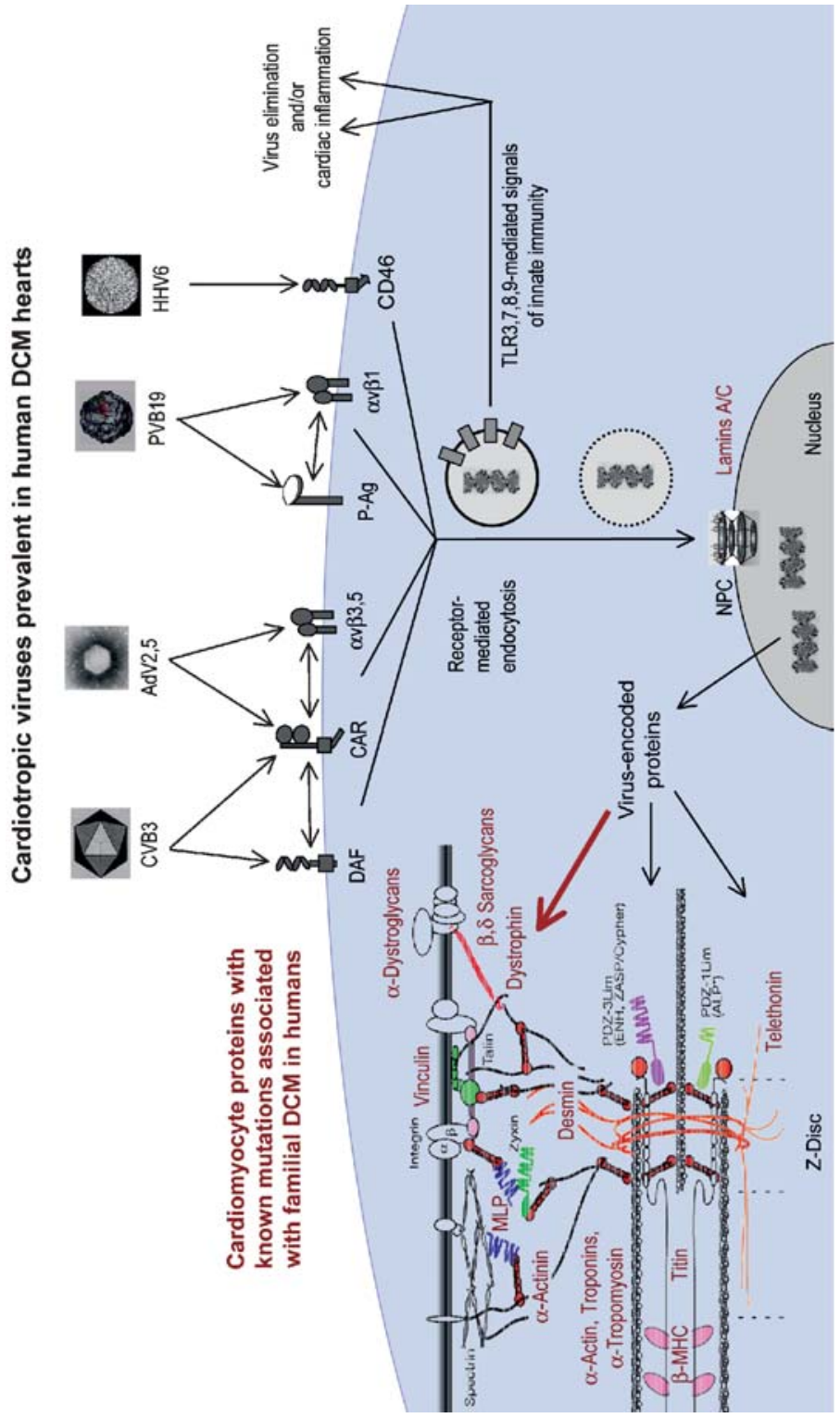




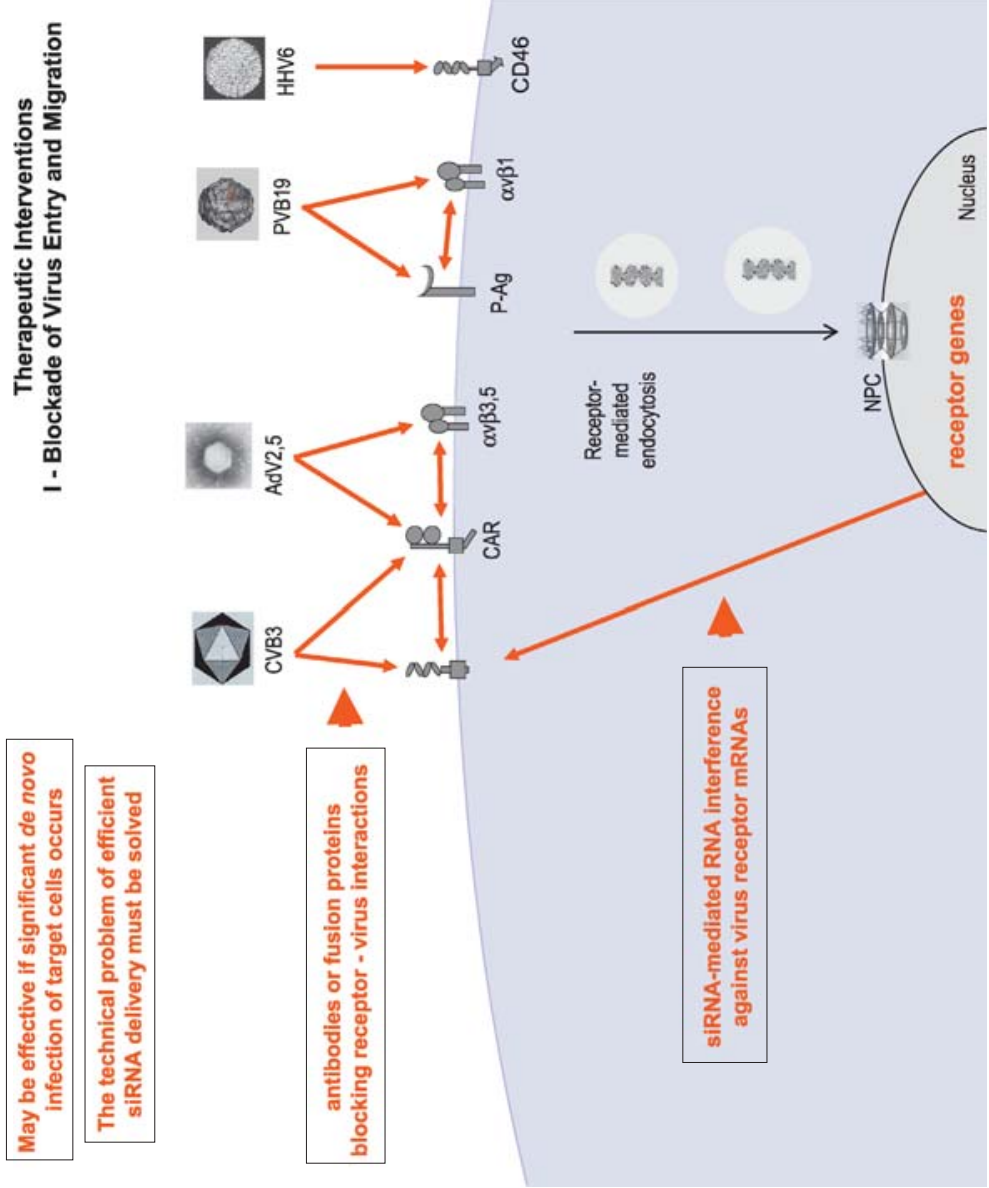

Fig. 2. Blockade of virus spreading and migration 

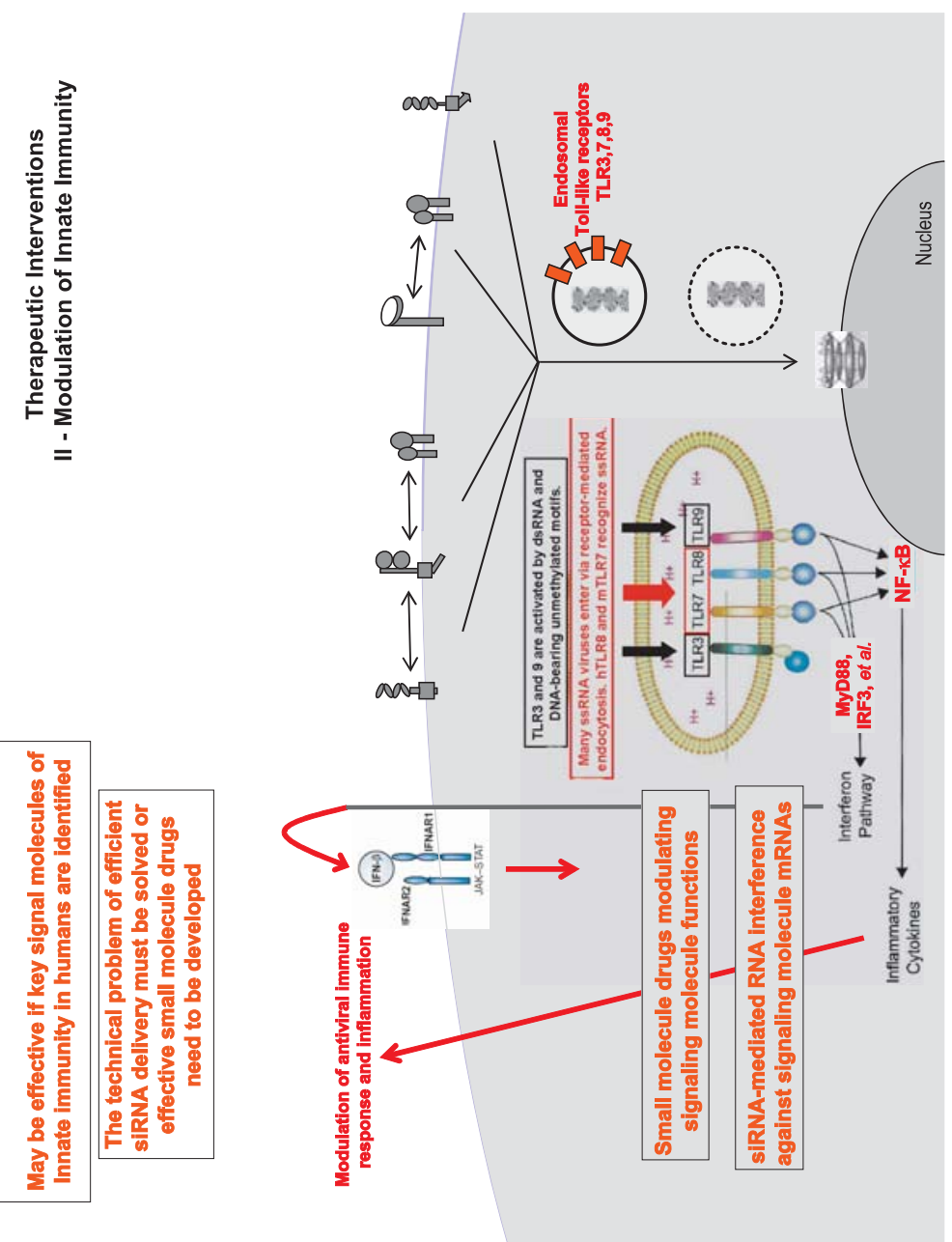

Fig. 3. Modulation of signaling pathways of innate immunity 

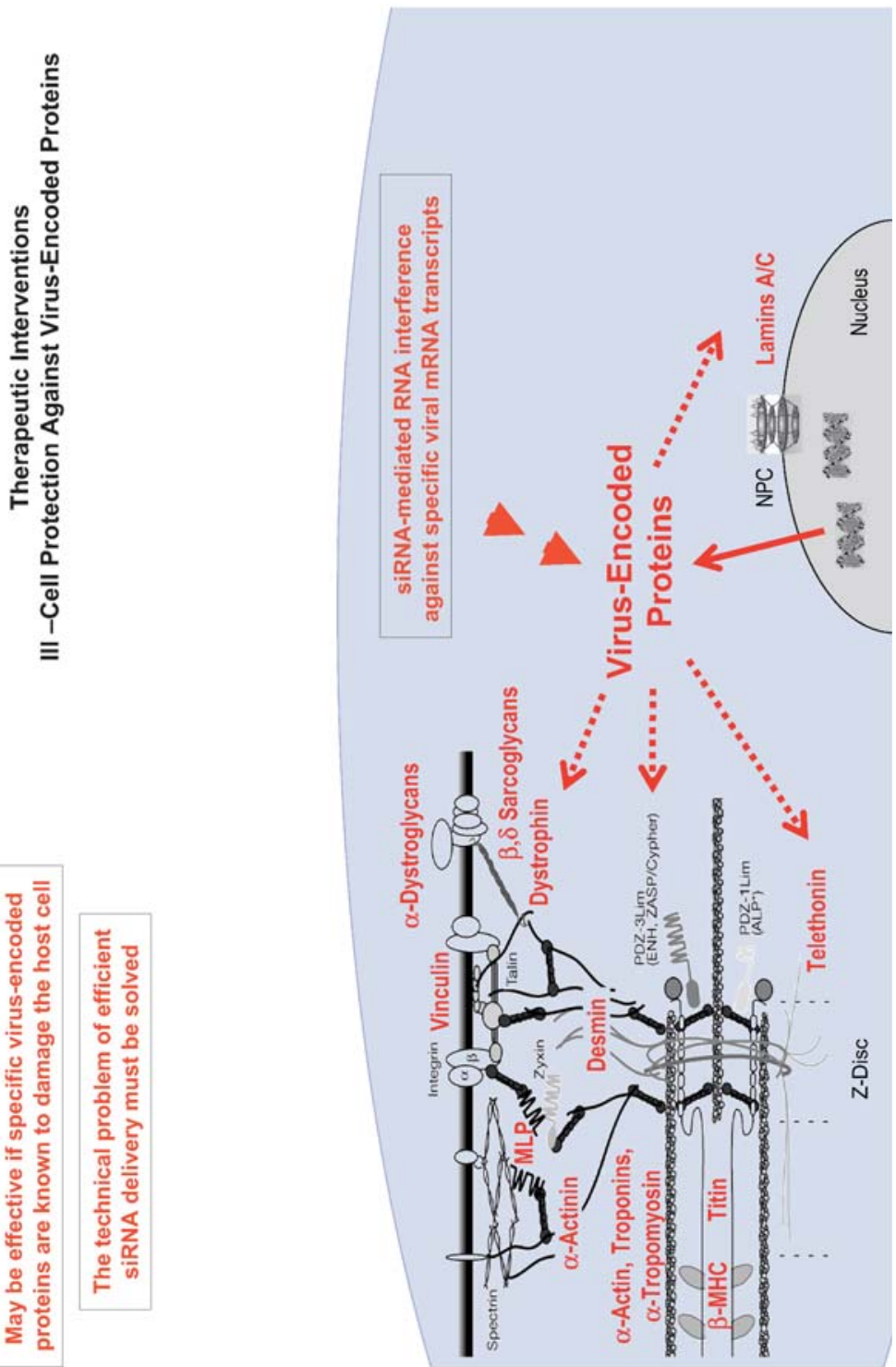

Fig. 4. Protection of host cells against virus-encoded proteins 
modulation of innate immune signaling may be useful in chronic infections, where persistent; overshooting inflammation is maintained by chronic signal pathway activation.

3. In the chronic phase of a viral cardiomyopathy, even when no new virus particles or viral genomes are synthesized and therefore no cell-to-cell virus spreading can possibly occur via receptor-mediated processes, there may still be chronic viral transcriptional activity damaging the host cells in a slow, insidious process. This may go unnoticed by the clinician unless major, irreversible cardiac damage occurred. One example illustrating this pathogenic principle is the cleavage of cellular dystrophin by the enteroviral protease $2 \mathrm{~A}$ encoded by CVB3 (Badorff et al. 1999; Xiong et al. 2002). Further proteins encoded by other cardiotropic viruses may also damage the infected heart even when synthesized at a low level only, but over long periods of time from cardiac-persistent viruses (Fig. 4). This may lead to an insidious decay of cardiac function proceeding unnoticed unless the patient is regularly and carefully monitored. "Silencing" of the respective viral genes, e.g., by RNA interference, constitutes a third target level for therapeutic interventions. Interventions of this type ("silencing strategies") may protect the chronically infected heart even after attempts at immune elimination of the virus-infected cells have remained unsuccessful.

\subsubsection{Therapeutic Targets (I): Virus Uptake Mechanisms}

\section{Virus Uptake Mechanisms}

Cellular virus entry, cell-to-cell spreading within the heart, and organto-organ migration within the host via the circulation are determined by virus receptor expression on target cells. The viral entry pathways into the human heart have not been fully delineated, but induction or inhibition of these pathways would decisively alter the individual susceptibility for cardiac viral infections. Induction of a common receptor (CAR) for coxsackieviruses and adenoviruses was observed in human DCM but not in further cardiomyopathies (Noutsias et al. 2001; Fechner et al. 2003). Receptor induction may open a path for infections with receptor-dependent viruses (EV/AdV) and contribute to dual infec- 
tions by the respective viruses, which are further influenced by distinct coreceptors. Another common receptor (CD46), shared by otherwise unrelated viruses (HHV6 and CAR-independent group B AdV), has been identified (Gaggar et al. 2003). It is currently unknown what genetic or environmental factors cause the dysregulation of CAR in DCM, but its induction may critically influence pathogenesis. Since virus genomes are prevalent in DCM hearts, genetic variants of virus receptors or their regulatory pathways could provide a molecular basis for important genome-environment interactions influencing the disease process.

Therapeutic targets at the receptor level as outlined in Fig. 2 may be expected to work (1) if there is significant de novo entry of virus into new target cells by cell-to-cell spreading within the heart, or by organ-to-organ migration within the host via the circulation; or (2) if it is possible block key entry mechanisms with high efficiency. Whereas there is significant virus migration in CVB3 myocarditis models in mice, little is known about the kinetics of cardiotropic viruses [PVB19, HHV6, CVB3, AdV, cytomegalovirus (CMV), EBV, HCV] detected in human chronic viral cardiomyopathy (Kühl et al. 2005). It is necessary to better characterize these kinetics to decide if anti-receptor approaches may have applications in the human disease too. The tools currently available to block or downregulate virus receptors are:

1. Antibodies or fusion proteins directed against the receptor protein and/or virus epitopes involved in virus binding and/or internalization. With respect to CVB3, the best-characterized human cardiotropic virus, there are interesting recent studies on the use of a soluble recombinant decay accelerating factor (DAF) in the form of an IgG1Fc-fusion protein (Yanagawa et al. 2003) or an analogous soluble CAR-Fc (Yanagawa et al. 2004). Importantly, however, these approaches work only in first few days after CVB3 infection. This a priori limits their possible use in humans to few cases of acute fulminant CVB3 myocarditis, but only if significant cell-to-cell migration of the CVB3 occurs in human too, similar to the situation in the animal model.

2. Antisense or decoy oligonucleotides (Yokoseki et al. 2001), which have been successfully employed in a myocarditis model. Short interfering RNAs (siRNAs) directed against an mRNA encoding a virus 
receptor have been used, e.g., for human immunodeficiency (HIV) inhibition (Zhou et al. 2004).

\subsubsection{Therapeutic Targets (II): Signaling Pathways of Innate Immunity}

\section{Signaling Pathways of Innate Immunity}

During their transport through the endosomal pathway, the RNA or DNA viral genome might engage certain TLRs $(3,7,8,9)$ linked to key signaling cascades of innate immunity (Ulevitch 2004). Genetic alterations of immune pathways decisively alter the disease course in viral infections. Thus, the mortality after CVB3 infection is very low in mice lacking MyD88, a key component of TLR-dependent innate immune signaling, as compared to wild-type animals. Mice are protected from CVB3 myocarditis by gene-targeted knockout of p56Lck, the Src family kinase essential for T cell activation (Liu et al. 2000). In contrast, CVB3 infection of INF- $\beta$-deficient mice results in excessive mortality as compared to controls (Deonarain et al. 2004). Extracellular signalregulated kinase 1 and 2 (ERK-1/2) is intense in the heart of myocarditissusceptible $\mathrm{A} / \mathrm{J}$ mice, in contrast to myocarditis-resistant $\mathrm{C} 57 \mathrm{BL} / 6$ mice. The ERK-1/2 response to CVB3 may thus contribute to differential host susceptibility too (Opavsky et al. 2002). In rat experimental autoimmune myocarditis a decoy oligonucleotide against nuclear factor (NF)- $\kappa$ B was highly efficient against the development of the disease (Yokoseki et al. 2001), but to our knowledge so far no analogous study of a viral heart disease has been published.

Therapeutic targets in virus-activated signaling cascades of innate immunity as outlined in Fig. 3 may be derived from recent work in genetic knockout animals infected with CVB3. Analogous to the improved survival upon infection in MyD88 (see Chap. 8, this volume) or p56Lck knockout animals (Liu et al. 2000), suppression of the respective genes by siRNA-mediated RNA interference should be investigated with respect to its therapeutic potential. Since MyD88 is at a key point of innate immune signaling, its suppression by siRNAs or a small molecule drug inhibiting MyD88 function may be highly efficient if inappropriate overshooting inflammatory reactions in the heart accompany the an- 
tiviral response. An analysis of MyD88 and other important immune signaling molecules in virus-infected human hearts seems worthwhile, since recent animal data suggest a high therapeutic potential. It is currently unknown if, in chronic human viral cardiomyopathy, similar innate immune signaling pathways as targeted in the specific CVB3 animal model are relevant. Other viruses are encountered in chronic human viral cardiomyopathy far more frequently (e.g., PVB19, HHV6) and the kinetics of the chronic human disease are grossly different from the animal model. It is therefore of interest to investigate by cardiac expression profiling (based on human cardiac biopsies), if each virus induces specific alterations of innate immune signaling, or if there is a common pattern across many different virus species. In the latter case, approaches directed at innate immunity may become therapeutically relevant for a large number of patients.

\subsubsection{Therapeutic Targets (III): Cellular Damage by Virus-Encoded Proteins}

\section{Cellular Damage by Virus-Encoded Proteins}

Beyond the proteins involved in cellular virus entry and antiviral defense, several structural proteins of the cardiomyocyte building the sarcomere, sarcolemma cytoskeleton, Z-bands, and intermediate filaments are known to be mutated in cases of familial DCM (Poller et al. 2005; Franz et al. 2001). No population-wide comprehensive mutation scanning has so far been published for these genes, and thus the full extent of their genetic variability is unknown. However, one pioneering experimental study has already demonstrated the extent to which such mutations may aggravate the cardiac damage caused by a virus, when CoxB3-infection led to grossly aggravated disease in dystrophin-deficient mice (Badorff et al. 1999; Xiong et al. 2002). The CoxB3-encoded enteroviral protease $2 \mathrm{~A}$ cleaves the cellular structural protein dystrophin not only in the dystrophin-deficient animals, but also in normal animals, although to a lesser extent. Most probably, a systematic search for further cellular sites vulnerable to attack by proteins which are encoded by human cardiotropic viruses will reveal further therapeutic targets. Protection of the respective vulnerable cellular proteins may, in principle, be achieved 
by siRNA-mediated suppression of the respective viral genes, or by specifically developed small-molecule drugs.

\section{Specific Therapeutic Tools - RNA Interference}

Whereas the very laborious development of small-molecule drugs blocking relevant virus-encoded proteins or their interaction site with a cellular protein must be done for every individual relevant protein, the application RNA interference by siRNAs is a more generalized approach. It has already been successfully employed against a number of different viruses both in vitro and in vivo (Zhou et al. 2004; Stevenson 2004; Ping et al. 2004; Dave and Pomerantz 2004; Gao et al. 2004; Zhang et al. 2004; Bhuyan et al. 2004; Takigawa et al. 2004; Kameoka et al. 2004; Wang et al. 2004; Lu et al. 2004; Li et al. 2004) including recent studies of CVB3 (Schubert et al. 2005), HHV6 (Yoon et al. 2004), and CMV inhibition in vitro (Wiebusch et al. 2004). One possible problem of siRNA therapy in vivo is the efficient transfer of the siRNA to all target cells. Recently, adeno-associated virus (AAV) vectors expressing siRNAs from specialized expression cassettes have been successfully used for various purposes (Pinkenburg et al. 2004; Grimm et al. 2005; Michel et al. 2005; Xia et al. 2004).

\subsection{Summary and Perspective}

As far as we currently understand chronic cardiac viral infections in humans, any therapeutic strategies inhibiting cellular virus uptake and migration (I) may only work if significant cell-to-cell traffic via receptormediated endocytosis of pathogenic virus still occurs in the chronic phase. At present we do not know if, e.g., cardiac reinfection from extracellular pools or intracardiac virus spreading occurs in the chronic disease in humans, and further clinical studies to solve this questions are warranted. Modulation of maladaptive innate immune signaling (II) was highly efficient in a CVB3 animal model. Further studies of the respective signaling pathways in humans should be performed, since the approach may possibly work across different virus species, thus providing a new generalized therapeutic principle. Finally, "silencing" (III) of viruses persisting in cardiac cells with very low transcriptional activity 
over long periods of time may inhibit the insidious damage to the heart as observed, e.g., in virus-positive DCM. If future molecular studies of cardiotropic viruses will identify virus-encoded proteins damaging key cellular proteins in cardiomyocytes or endothelial cells, this approach may gain major importance. In general, there are well-characterized CVB3 animal models, whereas there is a lack of animal models for other cardiotropic viruses and of data on their kinetics in chronic cardiac viral disease in humans. Clinical and animal studies addressing the latter issues are warranted, since they shall provide the basis for the development of improved therapies.

Acknowledgements. This publication has been supported by the Deutsche Forschungsgemeinschaft through Sonderforschungsbereich/Transregio 19.

\section{References}

Baboonian C, Davies M, Booth J, McKenna W (1997) Coxsackie B viruses and human heart disease. Curr Top Microbiol Immunol 223:31-52

Badorff C, Lee G, lamphear B, et al. (1999) Enteroviral protease 2A cleaves dystrophin: evidence of cytoskeletal disruption in an acquired cardiomyopathy. Nat Med 5:320-326

Bergelson J, Cunningham J, Droguett G, et al (1997) Isolation of a common receptor for coxsackie B viruses and adenoviruses 2 and 5. Science 275:13201323

Bhuyan PK, Kariko K, Capodici J, et al (2004) Short interfering RNA-mediated inhibition of herpes simplex virus type 1 gene expression and function during infection of human keratinocytes. J Virol 78:10276-10281

Bowles N, Richardson P, Olsen E, Archard L (1986) Detection of coxsackie-B virus-specific RNA sequences in myocardial biopsy samples from patients with myocarditis and dilated cardiomyopathy. Lancet 1:1120-1123

Chien K (2003) Genotype, phenotype: upstairs, downstairs in the family of cardiomyopathies. J Clin Invest 111:175-178

Dave RS, Pomerantz RJ (2004) Antiviral effects of human immunodeficiency virus type 1-specific small interfering RNAs against targets conserved in select neurotropic viral strains. J Virol 78:13687-13696

Deonarain R, Cerullo D, Fuse K, Liu PP, Fish EN (2004) Protective role for interferon-beta in coxsackievirus B3 infection. Circulation 110:3540-3543

Dörner A, Xiong D, Couch K, Yajima T, Knowlton K (2004) Alternatively spliced soluble Coxsackie-adenovirus receptors inhibit Coxsackievirus infection. J Biol Chem 279:18497-18503 
Fechner H, Noutsias M, Tschoepe C, et al (2003) Induction of coxsackievirusadenovirus-receptor expression during myocardial tissue formation and remodeling - identification of a cell-cell contact dependent regulatory mechanism. Circulation 107:876-882

Franz W, Muller O, Katus H (2001) Cardiomyopathies: from genetics to the prospect of treatment. Lancet 358:1627-1637

Frustaci A, Chimenti C, Calabrese F, Pieroni M, Thiene G, Maseri A (2003) Immunosuppressive therapy for active lymphocytic myocarditis: virologic and immunologic profile of responders versus non-responders. Circulation 107:857-863

Gaggar A, Shayakhmetov D, Lieber A (2003) CD46 is a cellular receptor for group B adenoviruses. Nat Med 9:1408-1412

Gao X, Wang H, Sairenji T (2004) Inhibition of Epstein-Barr virus (EBV) reactivation by short interfering RNAs targeting $\mathrm{p} 38$ mitogen-activated protein kinase or c-myc in EBV-positive epithelial cells. J Virol 78:11798-11806

Grimm D, Pandey K, Kay MA (2005) Adeno-associated virus vectors for short hairpin RNA expression. Methods Enzymol 392:381-405

Hertzog P, O'Neill L, Hamilton J (2003) The interferon in TLR signaling: more than just antiviral. Trends Immunol 24:534-539

Kameoka M, Nukuzuma S, Itaya A, et al. (2004) RNA interference directed against Poly(ADP-Ribose) polymerase 1 efficiently suppresses human immunodeficiency virus type 1 replication in human cells. J Virol 78:89318934

Kühl U, Pauschinger M, Schwimmbeck P, et al (2003a) Interferon- $\beta$ treatment eliminates cardiotropic viruses and improves left ventricular function in patients with myocardial persistence of viral genomes and left ventricular dysfunction. Circulation 107:2793-2798

Kühl U, Pauschinger M, Bock T, et al. (2003b) Parvovirus B19 infection mimicking acute myocardial infarction. Circulation 108:945-950

Kühl U, Pauschinger M, Noutsias M, Seeberg B, Bock T, Lassner D, Poller W, Kandolf R, Schultheiss HP (2005) High prevalence of viral genomes and multiple viral infections in the myocardium of adults with "idiopathic" left ventricular dysfunction. Circulation 111:887-893

Li G, Li XP, Peng Y, Liu X, Li XH (2004) Effect of inhibition of EBV-encoded latent membrane protein-1 by small interfering RNA on EBV-positive nasopharyngeal carcinoma cell growth. Di Yi Jun Yi Da Xue Xue Bao 24: 241-246

Liu P, Aitken K, Kong YY, Opavsky MA, Martino T, Dawood F, Wen WH, Kozieradzki I, Bachmaier K, Straus D, Mak TW, Penninger JM (2000) The tyrosine kinase p56lck is essential in coxsackievirus B3-mediated heart disease. Nat Med 6:429-434 
Lu A, Zhang H, Zhang X, et al. (2004) Attenuation of SARS coronavirus by a short hairpin RNA expression plasmid targeting RNA-dependent RNA polymerase. Virology 324:84-89

Matsumori A, Matoba Y, Sasayama S (1995) Dilated cardiomyopathy associated with hepatitis C virus infection. Circulation 92:2519-2525

Matsumori A, Yutani C, Ikeda Y, Kawai S, Sasayama S (2000) Hepatitis C virus from the hearts of patients with myocarditis and cardiomyopathy. Lab Invest 80:1137-1142

Michel U, Malik I, Ebert S, Bahr M, Kugler S (2005) Long-term in vivo and in vitro AAV-2-mediated RNA interference in rat retinal ganglion cells and cultured primary neurons. Biochem Biophys Res Commun 326:307-312

Murry C, Jerome K, Reichenbach D (2001) Fatal parvovirus myocarditis in a 5-year-old girl. Hum Pathol 32:342-345

Nigro G, Bastianon V, Colloridi V, et al (2000) Human parvovirus B19 infection in infancy associated with acute and chronic lymphocytic myocarditis and high cytokine levels: report of 3 cases and review. Clin Microbiol Infect 31:65-69

Noutsias M, Fechner H, Jonge Hd, et al (2001) Human coxsackie-adenovirusreceptor is co-localized with integrins avb3 and avb5 on the cardiomyocyte sarcolemma and upregulated in dilated cardiomyopathy - implications for cardiotropic viral infections. Circulation 104:275-280

Opavsky M, Martino T, Rabinovitch M, et al (2002) Enhanced ERK-1/2 activation in mice susceptible to coxsackievirus-induced myocarditis. J Clin Invest 109:1561-1569

Pauschinger M, Bowles N, Fuentes-Garcia J, et al. (1999a) Detection of adenoviral genome in the myocardium of adult patients with idiopathic left ventricular dysfunction. Circulation 99:1348-1354

Pauschinger M, Doerner A, Kuehl U, et al (1999b) Enteroviral RNA replication in the myocardium of patients with left ventricular dysfunction and clinically suspected myocarditis. Circulation 99:889-895

Ping YH, Chu CY, Cao H, Jacque JM, Stevenson M, Rana TM (2004) Modulating HIV-1 replication by RNA interference directed against human transcription elongation factor SPT5. J Acquir Immune Defic Syndr Hum Retrovirol 1:46

Pinkenburg O, Platz J, Beisswenger C, Vogelmeier C, Bals R (2004) Inhibition of NF-kappaB mediated inflammation by siRNA expressed by recombinant adeno-associated virus. J Virol Methods 120:119-122

Poller W, Kuhl U, Tschoepe C, Pauschinger M, Fechner H, Schultheiss HP (2005) Genome-environment interactions in the molecular pathogenesis of dilated cardiomyopathy. J Molec Med [Epub head of print 2 June 2005]

Rohayem J, Dinger J, Fischer R, Klingel K, Kandolf R, Rethwilm A (2001) Fatal myocarditis associated with acute parvovirus B19 and herpesvirus 6 coinfection. J Clin Microbiol 39:4585-4587 
Schubert S, Grunert H-P, Zeichhardt H, Werk D, Erdmann V, Kurreck J (2005) Maintaining inhibition: siRNA double expression vectors against coxsackieviral RNAs. J Mol Biol 346:457-465

Stevenson M (2004) Therapeutic potential of RNA interference. N Engl J Med 351:1772-1777

Takigawa Y, Nagano-Fujii M, Deng L, et al (2004) Suppression of hepatitis C virus replicon by RNA interference directed against the NS3 and NS5B regions of the viral genome. Microbiol Immunol 48:591-598

Ulevitch R (2004) Therapeutics targeting the innate immune system. Nat Rev Immunol 4:512-520

Wang Z, Ren L, Zhao X, et al. (2004) Inhibition of severe acute respiratory syndrome virus replication by small interfering RNAs in mammalian cells. J Virol 78:7523-7527

Why HJ, Meany BT, Richardson PJ, et al (1994) Clinical and prognostic significance of detection of enteroviral RNA in the myocardium of patients with myocarditis or dilated cardiomyopathy. Circulation 89:2582-2589

Wiebusch L, Truss M, Hagemeier C (2004) Inhibition of human cytomegalovirus replication by small interfering RNAs. J Gen Virol 85:179-184

Xia H, Mao Q, Eliason SL, et al. (2004) RNAi suppresses polyglutamineinduced neurodegeneration in a model of spinocerebellar ataxia. Nat Med 10:816-820

Xiong D, Lee G-H, Badorff C, et al. (2002) Dystrophin deficiency markedly increases enterovirus-induced cardiomyopathy: A genetic predisposition to viral heart disease. Nat Med 8:782-877

Yanagawa B, Spiller OB, Choy J, et al. (2003) Coxsackievirus B3-associated myocardial pathology and viral load reduced by recombinant soluble human decay-accelerating factor in mice. Lab Invest 83:75-85

Yanagawa B, Spiller OB, Proctor DG, et al (2004) Soluble recombinant coxsackievirus and adenovirus receptor abrogates coxsackievirus b3-mediated pancreatitis and myocarditis in mice. J Infect Dis 189:1431-1439

Yokoseki O, Suzuki J, Kitabayashi H, et al (2001) cis Element decoy against nuclear factor-kappaB attenuates development of experimental autoimmune myocarditis in rats. Circ Res 89:899-906

Yoon JS, Kim SH, Shin MC, et al. (2004) Inhibition of herpesvirus-6B RNA replication by short interference RNAs. J Biochem Mol Biol 37:383-385

Zhang XN, Xiong W, Wang JD, Hu YW, Xiang L, Yuan ZH (2004) siRNAmediated inhibition of HBV replication and expression. World J Gastroenterol 10:2967-2971

Zhou N, Fang J, Mukhtar M, Acheampong E, Pomerantz RJ (2004) Inhibition of HIV-1 fusion with small interfering RNAs targeting the chemokine coreceptor CXCR4. Gene Ther 11:1703-1712 